\title{
A SECOND ORDER LOGIC OF EXISTENCE ${ }^{1}$
}

\author{
NINO B. COCCHIARELLA
}

A. N. Prior in [9] has suggested an approach towards a second order logic of existence where, following medieval logicians, we distinguish "between predicates (like 'is red', 'is hard', etc.) which entail existence, and predicates (like 'is thought to be red', 'is thought of', etc.) which do not."2 Let us refer to attributes (including relational attributes) which are designated by the former kind of predicate as existence attributes, or for brevity, e-attributes. It is suggested then that ' $x$ exists' be defined as 'there is some e-attribute which $x$ possesses'. In what follows, this approach regarding the concept of existence is formalized semantically as well as syntactically, and a completeness theorem is established corresponding to the completeness (in a secondary sense, i.e., as including normal, nonstandard models) of standard second order logic (as formulated, for example, in Church [1]).

In [2] (cf. the abstracts [3] and [4]), the present author formalized a complete first order logic (with identity) of actual and possible objects which was there used in the formulation of a complete first order tense logic. ${ }^{3}$ The observation, which will be proved here, was made by Prior that in a second order logic with e-attributes quantification over actual objects can be appropriately defined so that every theorem of the first order logic of actual and possible objects becomes a theorem of second order logic with e-attributes. ${ }^{4}$ In effect, the definition (where $\phi$ is a formula, $\alpha$ is an individual variable, and $\pi$ is a 1-place predicate variable) is

$$
\stackrel{e}{\wedge} \alpha \phi=_{\mathrm{df}} \wedge \alpha[\stackrel{e}{\vee} \pi \pi(\alpha) \rightarrow \phi]
$$

or, in idiomatic English, 'every actual individual satisfies $\phi$ ' is construed as meaning 'every possible individual which possesses some e-attribute satisfies $\phi$ '.

Received April 5, 1968.

${ }^{1} \mathrm{I}$ am indebted to the referee for helpful suggestions in revisions of an earlier draft of this paper. For a more philosophical discussion of the present system, especially of the substitution free form of its axiom set, cf. [7].

2 p. 161.

3 In [5], completeness theorems in modal logic were also established utilizing this first order logic of actual and possible objects. In addition, completeness theorems for a logic of actual objects and a tense logic with quantification only over actual objects were also established, as well as subsequently a completeness theorem for a tense logic with quantification only over past and present objects.

4 op. cit., p. 162.

${ }^{5}$ It might be remarked, however, that the present formulation of second order logic with e-attributes is not exactly in the form which Prior has in mind. In [9, p. 161f ], Prior distinguishes quantification over e-attributes from quantification over attributes in general by means of different types of predicate variable. In the present version we do not make a distinction between types of predicate variables but rather introduce as primitive a new quantifier $\stackrel{e}{\wedge}$ for quantification over e-attributes (when followed by a predicate variable and for quantification over existing or actual objects when followed by an individual variable). 
§1. Terminology. As primitive logical constants, we take $\rightarrow$, the conditional sign; $\bigwedge$, the universal quantifier; and $\stackrel{\ell}{\wedge}$, the (restricted or) e-universal quantifier. Terms, formulas and other syntactical expressions are understood in the usual manner (cf. [6]), and for convenience we shall use ' $\phi$ ', ' $\psi$ ', ' $\chi$ ' to refer to formulas, ' $\alpha$ ', ' $\beta$ ', ' $\gamma$ ' to refer to individual variables, ' $\pi$ ', ' $\rho$ ', ' $\sigma$ ', ' $\tau$ ' to refer to predicate expressions, ' $\zeta$ ', ' $\eta$ ', ' $\xi$ ' to refer to terms, and ' $\mu$ ', ' $\nu$ ' to refer to both individual and predicate variables. Regarding proper substitution of formulas for predicate variables, we adopt the definition given in Church [1, p. 192f]. However, we shall utilize the notation.

in place of Church's notation

$$
\phi\left[\begin{array}{c}
\pi\left(\alpha_{0}, \cdots, \alpha_{n-1}\right) \\
\psi
\end{array}\right]
$$

$$
\left.\mathrm{S}^{\pi\left(\alpha_{0}, \cdots, \alpha_{n-1}\right)} \phi\right|^{6}
$$

\$2. The semantics of second order logic with e-attributes. In describing the model-theoretic semantics of a logic in which we wish to distinguish actual objects from possibilia in general, it is appropriate that a distinction be made between the universe of actual (existing) objects of a given model and the (usually) wider domain of discourse or set of possibilia of that model. In [2] and [3], such a notion of a model for the first order logic of actual and possible objects there constructed was characterized and in a way so as to preserve the essential content of Tarski's wellknown procedures in this area. We retain that notion here and, of course, develop or expand upon it for purposes of second order logic with e-attributes. Accordingly, where $L$ is a language, we say that $\mathfrak{A}$ is a model suited to $L$, or for brevity, is an $L$-model, if and only if there are sets $A, B, R$ such that $\mathfrak{U}=\langle A, B, R\rangle, A \subseteq B$ (i.e., the universe of actual objects of $\mathfrak{A}$ is included in the domain of discourse or set of possibilia of $\mathfrak{U}$ ), $B \neq 0$ (i.e., $\mathfrak{A}$ is not devoid of possibilia though it may have no actual objects), $R$ is a function with $L$ as its domain, and for all $n, \pi, \delta$, (i) if $n \in \omega$ and $\pi$ is an $n$-place predicate constant in $L$, then $R(\pi) \subseteq B^{n}$, and (ii) if $n \in \omega$ and $\delta$ is an $n$-place operation constant in $L$, then $R(\delta) \in B^{B^{n}} \cdot{ }^{7}$ We say that $\mathfrak{A}$ is a model if $\mathfrak{A}$ is an $L$-model for some language $L$. By the universe of (actual objects of) a model $\mathfrak{A}$, in symbols $\mathscr{U}_{\mathfrak{Q}}$, the set of possibilia relative to $\mathfrak{A}$, in symbols $\mathscr{P}_{\mathfrak{v}}$, and the language of $\mathfrak{A}$, in symbols $L_{\mathfrak{q}}$, we understand the sets $A, B$ and the domain of $R$, respectively, where $\mathfrak{A}=\langle A, B, R\rangle$. If $\theta \in L_{\mathfrak{r}}$, we set $\theta_{\mathfrak{l}}=R(\theta)$.

Following Henkin in [8], we say, where $L$ is a language, that $\mathfrak{B}$ is a secondary

${ }^{6}$ Church's definition must of course be amended to recognize that occurrences of predicate and individual variables may be bound by either of our two quantifiers, $\wedge, \stackrel{e}{\wedge}$.

7 As usual, we take $\omega$ to be the set of natural numbers and read ' $n \in \omega$ ' as ' $n$ is a natural number'. In addition, where $X$ and $Y$ are sets, $X^{Y}$ is understood to be the set of functions with $Y$ as domain and with ranges included in $X$. We understand each natural number to be the set of natural numbers less than it and understand an $n$-termed sequence or an $n$-tuple, where $n \in \omega$, to be a function with $n$ as its domain. Consequently, where $n \in \omega, B^{n}$ is the set of all $n$-termed sequences whose constituents are in $B$. 
$L$-model if and only if there are an $L$-model $\mathfrak{A}$ and an $\omega$-indexed family $\left\langle\mathbf{F}_{n}\right\rangle_{n \in \omega}$ such that $\mathfrak{B}=\left\langle\mathfrak{A},\left\langle\mathbf{F}_{n}\right\rangle_{n \in \omega}\right\rangle$ and for each $n \in \omega, \mathbf{F}_{n}$ is a subset of the set of all subsets of $\mathscr{P}_{\mathfrak{r}}$ (i.e., every member of $\mathbf{F}_{n}$ is a subset of $\mathscr{P}_{\mathfrak{q}}$ ). We understand $\mathfrak{B}$ to be a secondary model, or for brevity a 2 -model, if $\mathfrak{B}$ is a secondary $L$-model for some language $L$. Where $\mathfrak{B}=\left\langle\mathfrak{A},\left\langle\mathbf{F}_{n}\right\rangle_{n \in \omega}\right\rangle$ and $\mathfrak{B}$ is a 2 -model, we set $\mathscr{U}_{\mathfrak{Q}}=\mathscr{U}_{\mathfrak{q}}$, $\mathscr{P}_{\mathfrak{P}}=\mathscr{P}_{\mathfrak{U}}$ and $L_{\mathfrak{P}}=L_{\mathfrak{r}}$.

We say that $a$ is an assignment in a 2 -model $\left\langle\mathfrak{A},\left\langle\mathbf{F}_{n}\right\rangle_{n \in \omega}\right\rangle$ if $a$ is a function whose domain is the set of variables (both individual and predicate) and which is such that (1) for each individual variable $\alpha, a(\alpha) \in \mathscr{P}_{\mathrm{q}}$, and (2) for each $n \in \omega$ and for each $n$ place predicate variable $\pi, a(\pi) \in \mathbf{F}_{n}$. If $a$ is such an assignment of values to variables, we understand $a\left(\begin{array}{l}\mu \\ x\end{array}\right)$, where $\mu$ is a predicate or individual variable, to be that assignment (of values to variables) which is identical to $a$ in all respects except (at most) in its assigning $x$ to $\mu$.

The notion of the value of a term $\zeta$ or of a predicate expression $\pi$ of a language $L$ with respect to a secondary $L$-model $\mathfrak{A}$ and an assignment $a$ in $\mathfrak{A}$, in symbols $\operatorname{Val}(\zeta, \mathfrak{A}, a)$ or $\operatorname{Val}(\pi, \mathfrak{A}, a)$, is characterized recursively as follows: (1) if $\mu$ is an individual or a predicate variable, then $\operatorname{Val}(\mu, \mathfrak{A}, a)=a(\mu),(2)$ if $\pi$ is a predicate constant in $L$, then $\operatorname{Val}(\pi, \mathfrak{A}, a)=\pi_{\mathfrak{U}}$, and (3) if $n \in \omega, \delta$ is an $n$-place operation constant in $L$, and $\zeta_{0}, \cdots, \zeta_{n-1}$ are terms of $L$, then $\operatorname{Val}\left(\delta\left(\zeta_{0}, \cdots, \zeta_{n-1}\right), \mathfrak{A}, a\right)=$ $\delta_{\mathfrak{r}}\left(\operatorname{Val}\left(\zeta_{0}, \mathfrak{X}, a\right), \cdots, \operatorname{Val}\left(\zeta_{n-1}, \mathfrak{A}, a\right)\right)$.

Where $\mathfrak{A}$ is a 2-model and $a$ is an assignment in $\mathfrak{A}$, we characterize the notion of satisfaction of an arbitrary formula of $L_{\mathfrak{U}}$ (by $a$ in $\mathfrak{X}$ ) by the following recursive clauses: (1) if $n \in \omega, \pi$ is an $n$-place predicate expression of $L_{\mathscr{Q}}$, and $\zeta_{0}, \cdots, \zeta_{n-1}$ are terms of $L_{\mathfrak{U}}$, then $a$ satisfies $\pi\left(\zeta_{0}, \cdots, \zeta_{n-1}\right)$ in $\mathfrak{X}$ if and only if $\left\langle\operatorname{Val}\left(\zeta_{0}, \mathfrak{A}, a\right), \cdots\right.$, $\left.\operatorname{Val}\left(\zeta_{n-1}, \mathfrak{A}, a\right)\right\rangle \in \operatorname{Val}(\pi, \mathfrak{A}, a) ;(2)$ if $\phi, \psi$ are formulas of $L$, then $a$ satisfies $(\phi \rightarrow \psi)$ in $\mathfrak{A}$ if and only if either $a$ does not satisfy $\phi$ in $\mathfrak{A}$ or $a$ satisfies $\psi$ in $\mathfrak{A} ;(3)$ if $\phi$ is a formula of $L_{\mathfrak{U}}$ and $\alpha$ is an individual variable, then $a$ satisfies $\Lambda \propto \phi$ in $\mathfrak{U}$ if and only if for all $x \in \mathscr{P}_{\mathfrak{u}}, a\left(\begin{array}{l}\alpha \\ x\end{array}\right)$ satisfies $\phi$ in $\mathfrak{X}$; (4) if $\phi$ is a formula of $L_{\mathfrak{U}}$ and $\alpha$ is an individual variable, then $a$ satisfies $\bigwedge^{e} \alpha \phi$ in $\mathfrak{A}$ if and only if for all $x \in \mathscr{U}_{\mathfrak{l}}, a\left(\begin{array}{l}\alpha \\ x\end{array}\right)$ satisfies $\phi$ in $\mathfrak{A}$; (5) if $\phi$ is a formula of $L_{\mathfrak{l}}, n \in \omega$, and $\pi$ is an $n$-place predicate variable, then $a$ satisfies $\wedge \pi \phi$ in $\mathfrak{A}$ if and only if for all $F \in \mathbf{F}_{n}, a\left(\begin{array}{l}\pi \\ F\end{array}\right)$ satisfies $\phi$ in $\mathfrak{A}$; and (6) if $\phi$ is a formula of $L_{\mathfrak{\vartheta}}, n \in \omega$, and $\pi$ is an $n$-place predicate variable, then $a$ satisfies $\bigwedge^{e} \pi \phi$ in $\mathfrak{A}$ if and only if for all $F \in \mathbf{F}_{n}$, if $F \subseteq \mathscr{U}_{\mathfrak{u}}^{n}$, then $a\left(\begin{array}{l}\pi \\ F\end{array}\right)$ satisfies $\phi$ in $\mathscr{U}$. If $\mathfrak{A}$ is a 2-model, then a formula of $L_{\mathfrak{Q}}$ is said to be true in $\mathfrak{A}$ if it is satisfied by every assignment in $\mathfrak{A}$.

In regard to the notion of secondary validity (logical truth) a certain rather natural restriction must be imposed on 2-models to obtain what we, following Church, shall call the normal 2-models, a restriction which in effect stipulates that in order for a 2-model $\left\langle\mathfrak{A},\left\langle\mathbf{F}_{n}\right\rangle_{n \in \omega}\right\rangle$ to be relevant or normal, each $\mathbf{F}_{n}$, for $n \in \omega$, must be closed in a rather obvious way. What is desired is that we restrict our considerations to those 2 -models $\left\langle\mathfrak{A},\left\langle\mathbf{F}_{n}\right\rangle_{n \in \omega}\right\rangle$ which are such that whenever a condition can 
be specified by means of a formula $\phi$ of $L_{\mathfrak{u}}$ with, say, $n$ distinct free individual variables, then there exists in $\mathbf{F}_{n}$ an ( $n$-place) attribute $F$ (i.e., a set $F \subseteq \mathscr{P}_{2}$ ) such that all and only those $n$-tuples of (possible) individuals of $\mathfrak{U}$ possess (belong to) $F$ that satisfy the condition $\phi$. This rather obviously desired restriction can be captured as follows. We shall say that a 2 -model $\mathfrak{B}$ is normal if and only if for each formula $\phi$ of $L_{\mathscr{B}}$, the formula $\bigvee \pi \wedge \alpha_{0} \cdots \wedge \alpha_{n-1}\left[\pi\left(\alpha_{0}, \cdots, \alpha_{n-1}\right) \leftrightarrow \phi\right]$ is true in $\mathscr{B}$, where $n \in \omega, \pi$ is an $n$-place predicate variable which does not occur free in $\phi$, and $\alpha_{0}, \cdots, \alpha_{n-1}$ are all the distinct individual variables occurring free in $\phi{ }^{8}$

Accordingly, $\phi$ is said to be secondarily valid, or for brevity, 2-valid, if and only if there is a language $L$ such that $\phi$ is a formula of $L$ and $\phi$ is true in every normal secondary $L$-model.

\$3. A substitution free axiom set for second order logic with e-attributes. In what follows we present a complete (with respect to 2-validity) formulation of second order logic with e-attributes by means of an axiom set whose characterization does not require the notion of proper substitution of either a term for an individual variable or of a formula for a predicate variable.

Definition. A formula $\theta$ is a (second order) logical axiom if and only if there are natural numbers $n, k$, individual variables $\alpha, \beta_{0}, \cdots, \beta_{n-1}, \gamma_{0}, \cdots, \gamma_{k-1}$, predicate variables $\pi, \rho, \sigma, \tau$, a predicate or individual variable $\mu$, terms $\zeta$, $\eta$, and formulas $\phi, \psi, \chi$ such that $\theta$ is a generalization of one of the following formulas:

(A1) $\phi \rightarrow(\psi \rightarrow \phi)$

(A2) $[\phi \rightarrow(\psi \rightarrow \chi)] \rightarrow[(\phi \rightarrow \psi) \rightarrow(\phi \rightarrow \chi)]$

(A3) $(\sim \phi \rightarrow \sim \psi) \rightarrow(\psi \rightarrow \phi)$

(A4) $\wedge \mu(\phi \rightarrow \psi) \rightarrow(\bigwedge \mu \phi \rightarrow \wedge \mu \psi)$

(A5) $\phi \rightarrow \wedge \mu \phi$,

where $\mu$ does not occur free in $\phi$,

(A6) $\vee \pi \wedge \beta_{0} \cdots \wedge \beta_{n-1}\left[\pi\left(\beta_{0}, \cdots, \beta_{n-1}\right) \leftrightarrow \phi\right]$,

where $\beta_{0}, \cdots, \beta_{n-1}$ are all the distinct individual variables that occur free in $\phi$ and $\pi$ is an $n$-place predicate variable which does not occur free in $\phi$,

(A7) $\bigvee \alpha \wedge \rho[\rho(\alpha) \rightarrow \rho(\zeta)]$, where $\alpha$ is an individual variable which does not occur in $\zeta$,

(A8) $\wedge \rho[\rho(\zeta) \rightarrow \rho(\eta)] \rightarrow(\phi \rightarrow \psi)$, where $\phi, \psi$ are atomic formulas, and $\psi$ is obtained from $\phi$ by replacing an occurrence of $\zeta$ with an occurrence of $\eta$,

\footnotetext{
${ }^{8}$ This characterization of normal 2-models differs somewhat from that given by Church in $[1$, p. 308], as well as his equivalent alternative formulation on p. 316. (Church's latter formulation in effect would not require that $\alpha_{0}, \cdots, \alpha_{n-1}$ be all the distinct individual variables with free occurrences in $\phi$, but only that $\alpha_{0}, \cdots, \alpha_{n-1}$ be distinct individual variables whether they occur in $\phi$ or not.) Nevertheless, it can easily be shown to be equivalent to either of Church's characterizations (with the understanding that we have a wider notion of formulahood involved), and it has the virtue of being somewhat more obvious or perspicacious for obtaining the closure conditions desired, especially in the light of the notion of definability. (This equivalence for standard formulas, i.e., those in which $\stackrel{e}{\wedge}$ does not occur, follows directly from Theorem 1 below and the main result established in [6].)
} 
(A9) $\bigwedge^{e} \sigma(\phi \rightarrow \psi) \rightarrow\left(\AA^{\circ} \sigma \phi \rightarrow \AA^{\circ} \sigma \phi\right)$,

(A10) $\wedge \sigma \phi \rightarrow \stackrel{i}{\wedge} \sigma \phi$,

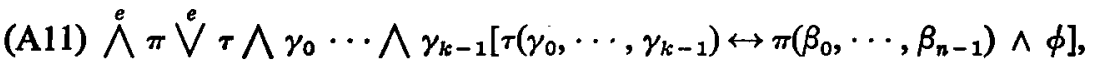

where $\pi$ is an $n$-place predicate variable which does not occur free in $\phi, \tau$ is a $k$-place predicate variable distinct from $\pi$ and which does not occur free in $\phi$, $\beta_{0}, \cdots, \beta_{n-1}$ are all the distinct individual variables which occur free in $\phi$, and $\gamma_{0}, \cdots, \gamma_{k-1}$ are pairwise distinct individual variables such that $\left\{\gamma_{0}, \cdots, \gamma_{k-1}\right\} \subseteq$ $\left\{\beta_{0}, \cdots, \beta_{n-1}\right\}$,

(A12) $\stackrel{e}{\bigvee} \pi \wedge \beta_{0} \cdots \wedge \beta_{n-1}\left[\pi\left(\beta_{0}, \cdots, \beta_{n-1}\right) \leftrightarrow \bigvee^{e} \tau \tau\left(\beta_{0}, \cdots, \beta_{n-1}\right)\right]$,

where $\beta_{0}, \cdots, \beta_{n-1}$ are pairwise distinct individual variables,

$$
\stackrel{e}{\wedge} \beta_{0} \cdots \stackrel{e}{\wedge} \beta_{n-1} \phi \leftrightarrow \wedge \beta_{0} \cdots \wedge \beta_{n-1}\left[\bigvee^{e} \pi \pi\left(\beta_{0}, \cdots, \beta_{n-1}\right) \rightarrow \phi\right] .
$$

We shall have only one inference rule in the present system, viz., modus ponens. Derivation of a formula $\phi$ from a set $\Gamma$ of formulas is understood in the usual way, and we say $\Gamma$ yields $\phi$, in symbols $\Gamma \vdash \phi$, if $\phi$ is derivable from $\Gamma$. If $\Gamma$ is empty, we write ' $\vdash \phi$ ' for ' $\Gamma \vdash \phi$ ' and say that $\phi$ is a theorem (of second order logic with e-attributes) if $r \phi$.

In [6], it was shown that (A1)-(A8) characterize a complete (with respect to 2-validity) axiom set for standard second order logic. A completeness theorem (with respect to 2-validity) for the extension of the notion of standard formula to that of formula (as defined here, i.e., as allowing occurrences of $\stackrel{e}{\Lambda}$ ) of course requires axioms in addition to (A1)-(A8).

THEOREM 1. If $\phi$ is a theorem of second order logic with e-attributes, then $\phi$ is secondarily valid.

Proof. It is clear from the definition of satisfaction (and hence of truth) that every logical axiom of any of the forms (A1)-(A5), (A7)-(A10) is true in any secondary model (in the language of which it is formulable) and that modus ponens preserves truth in every model. Moreover, that every axiom of the form (A6) is 2-valid is an immediate consequence of the definition of 2-validity. It remains only to consider axioms of the forms (A11)-(A13); but as the proofs of 2-validity are straightforward we will only detail the proof of (A12).

Suppose that $\psi$ is an instance of (A12) formulable in a normal 2-model $\mathfrak{B}=$ $\left\langle\mathfrak{A},\left\langle\mathbf{F}_{n}\right\rangle_{n \in \omega}\right\rangle$. Then $\psi=\stackrel{e}{V} \pi \wedge \beta_{0} \cdots \wedge \beta_{n-1}\left[\pi\left(\beta_{0}, \cdots, \beta_{n-1}\right) \leftrightarrow \bigvee^{e} \tau \tau\left(\beta_{0}, \cdots, \beta_{n-1}\right)\right]$. Assume now that $a$ is an assignment in $\mathfrak{B}$. Then, since $\mathfrak{O}$ is normal, $a$ satisfies $\bigvee \pi \wedge \beta_{0} \cdots \wedge \beta_{n-1}\left[\pi\left(\beta_{0}, \cdots, \beta_{n-1}\right) \leftrightarrow \bigvee^{e} \tau\left(\beta_{0}, \cdots, \beta_{n-1}\right)\right]$ in $\mathfrak{B}$, this formula being an instance of (A6). Accordingly, there is an $F \in \mathbf{F}_{n}$ such that for all $x_{0}, \cdots, x_{n-1} \in \mathscr{P}_{\mathfrak{g}},\left\langle x_{0}, \cdots, x_{n-1}\right\rangle \in F$ if and only if $a\left(\begin{array}{c}\pi \beta_{0} \cdots \beta_{n-1} \\ F x_{0} \cdots x_{n-1}\end{array}\right)$ satisfies 
$\bigvee_{\tau \tau}^{e}\left(\beta_{0}, \cdots, \beta_{n-1}\right)$ in $\mathfrak{B}$, i.e., if and only if there is a $G \in \mathbf{F}_{n}$ such that $G \subseteq \mathscr{U}^{n}$ and $\left\langle x_{0}, \cdots, x_{n-1}\right\rangle \in G$; from which it follows that any $n$-tuple in $F$ is an $n$-tuple in some subset of $\mathscr{U}_{\mathbb{*}}^{n}$, and therefore that $F \subseteq \mathscr{Q}_{\mathrm{P}}^{n}$. Consequently, $a$ satisfies $\bigvee^{e} \pi \wedge \beta_{0} \cdots \wedge \beta_{n-1}\left[\pi\left(\beta_{0}, \cdots, \beta_{n-1}\right) \leftrightarrow \bigvee^{e} \tau \tau\left(\beta_{0}, \cdots, \beta_{n-1}\right)\right]$ in $\mathscr{B}$. We conclude then that every instance of (A12) is 2-valid.

\$4. Some theorems of second order logic with e-attributes. In order to prove that our present axiomatic formulation is complete (with respect to 2-validity), we need to establish some useful lemmas, the most important of which are the specification principles regarding each kind of quantification. Where proofs are not given it is understood that they proceed exactly as in the analogous standard situation. We avoid writing obvious lemmas such as the deduction theorem. We utilize our convention of having specific groups of Greek letters for the different kinds of expression in what follows by not bothering to specify in each case the kind of expression involved.

We presuppose the notion of a tautology or tautologous formula without going into the definition here. Because of (A1)-(A3) and the completeness of sentential logic, we have the following lemma.

LEMMA 1. If $\phi$ is a tautologous formula, then $\vdash \phi$.

LEMMA 2. If $\Gamma \vdash \phi$ and $\Gamma \vdash(\phi \rightarrow \psi)$, then $\Gamma \vdash \psi$.

LEMMA 3. If $\Gamma \vdash \phi$ and $\mu$ is a predicate or individual variable which does not occur free in any member of $\Gamma$, then $\Gamma \vdash \wedge \mu \phi$.

LEMMA 4. If $\Gamma \vdash \phi\left[\begin{array}{l}\alpha \\ \zeta\end{array}\right]$ and $\zeta$ does not occur in any member of $\Gamma$, then $\Gamma \vdash \wedge \alpha \phi$.

LEMMA 5. If $\Gamma \vdash \phi\left[\begin{array}{l}\pi \\ \rho\end{array}\right]$ and $\rho$ does not occur in any member of $\Gamma$, then $\Gamma \vdash \wedge \pi \phi$.

LEMMA 6. If $\Gamma \vdash \phi$ and $\mu$ is a predicate or individual variable which does not occur free in any member of $\Gamma$, then $\Gamma \vdash \stackrel{\wedge}{\mu} \phi$.

Proof. Lemma 6 follows directly from (A10), (A13) and Lemmas 2 and 3. (Q.E.D.)

LEMMA 7. If $\mu$ is a predicate or individual variable which does not occur free in $\phi$, then $\vdash \phi \rightarrow \AA \mu \phi$.

PRoof. Lemma 7 follows trivially from (A5), (A10), (A13) and Lemmas 1, 2 and 3. (Q.E.D.)

LEMMA 8. $\vdash \wedge \alpha \phi \rightarrow \stackrel{e}{\wedge} \alpha \phi$.

PROOF. Lemma 8 is an immediate consequence of (A13) and Lemmas 1 and 2. (Q.E.D.)

LEMMA 9. $\vdash \stackrel{e}{\wedge} \alpha(\phi \rightarrow \psi) \rightarrow\left(\bigwedge^{e} \alpha \phi \rightarrow(\stackrel{e}{\wedge} \alpha \psi)\right.$.

Proof. Lemma 9 follows directly from (A2), (A4), (A13) and Lemmas 1, 2 and 3. (Q.E.D.)

We note that Lemmas 8 and 9 are two of the axioms of the first order logic of actual and possible objects formulated in [2] and [3]. The proofs for Lemmas 10, 11 , and 12 are similar to the proofs of their analogues in the system of [6]. 
LEMMA 10. If $\psi$ is obtained from $\phi$ by replacing a free occurrence of $\zeta$ by a free occurrence of $\eta$, then $\vdash \wedge \pi[\pi(\zeta) \rightarrow \pi(\eta)] \rightarrow(\phi \rightarrow \psi)$ and $\vdash \wedge \pi[\pi(\zeta) \rightarrow \pi(\eta)] \rightarrow(\psi \rightarrow \phi)$.

For convenience let us use $\equiv$ for the sign of identity, definable (in the metalanguage) as follows:

$$
\zeta \equiv \eta={ }_{\mathrm{dr}} \wedge \pi[\pi(\zeta) \rightarrow \pi(\eta)]
$$

(where $\pi$ is, say, the first 1-place predicate variable). Lemma 10 , of course, establishes the general form of Leibniz' law as a theorem, i.e., $t \zeta \equiv \eta \rightarrow(\phi \rightarrow \psi)$, where $\psi$ is obtained from $\phi$ by replacing a free occurrence of $\zeta$ by a free occurrence of $\eta$.

LEMMA 11. If $\alpha$ does not occur in $\zeta$ and $\psi$ is obtained from $\phi$ by proper substitution of $\zeta$ for $\alpha$, then $\vdash \wedge \alpha \phi \rightarrow \psi$.

Lemma 12, to follow, is our general specification principle for possibilia.

LEMMA 12. If $\psi$ is obtained from $\phi$ by proper substitution of $\zeta$ for $\alpha$, then $\vdash \wedge \alpha \phi \rightarrow \psi$.

Lemma 13, to follow, is one variant of our specification principle for actual objects.

LEMMA 13. If $\psi$ is obtained from $\phi$ by proper substitution of $\zeta$ for $\alpha$, then $\vdash \bigvee^{e} \pi(\zeta) \rightarrow(\grave{\wedge} \alpha \phi \rightarrow \psi)$.

Proof. Assume the hypothesis. Then,

$\vdash \wedge \alpha\left[\bigvee^{e} \pi \pi(\alpha) \rightarrow \phi\right] \rightarrow\left(\bigvee^{e} \pi \pi(\zeta) \rightarrow \psi\right) \quad$ by Lemma 12 ,

$\vdash \bigwedge^{e} \alpha \phi \rightarrow\left(\vee^{e} \pi \pi(\zeta) \rightarrow \psi\right)$

by (A13) and Lemmas 1 and 2, and therefore

$H \dot{\vee} \pi \pi(\zeta) \rightarrow(\stackrel{\complement}{\Lambda} \alpha \phi \rightarrow \psi)$

by Lemmas 1 and 2. (Q.E.D.)

The formula $\bigvee^{e} \pi \pi(\zeta)$ asserts (with respect to a 2-model $\mathfrak{B}$ ) that the referent of $\zeta$ (in $\mathfrak{B}$ ) possesses some e-attribute, and therefore that the referent of $\zeta$ (in $\mathfrak{B}$ ) is an actual object (of $\mathfrak{B}$ ), i.e., that the referent of $\zeta$ exists (as an actual object of $\mathfrak{B}$ ). Defining the predicate $E$ ! for existence is of course straightforward:

$$
E !(\alpha)={ }_{\text {ar }} \bigvee \pi \pi(\alpha)
$$

In the first order logic (with identity) of actual and possible objects of [2] and [3], assertion of the existence of the referent of $\zeta$ (in a model $\mathfrak{A}$ ) was captured by means of the formula $\bigvee^{e} \alpha \alpha \equiv \zeta$, which in the higher order context is understood to be an abbreviation of $\bigvee^{e} \wedge \wedge \pi[(\alpha) \rightarrow \pi(\zeta)]$. Accordingly, an alternative definition of $E$ ! is

$$
E !(\alpha)={ }_{\mathrm{dr}} \bigvee^{e} \beta \wedge \pi[\pi(\beta) \rightarrow \pi(\alpha)]
$$

The following two lemmas, of which the proofs are straightforward, establish the equivalence of these two alternative definitions of existence.

LEMMA 14. If $\alpha, \beta$ are distinct individual variables, then $\vdash^{e} \beta \wedge \pi[\pi(\beta) \rightarrow \pi(\alpha)] \rightarrow$ $\bigvee^{e} \pi \pi(\alpha)$.

LEMMA 15. If $\alpha, \beta$ are distinct individual variables, then $\vdash^{e} \pi \pi(\alpha) \rightarrow \vee^{e} \beta \wedge$ $\pi[\pi(\beta) \rightarrow \pi(\alpha)]$. 
LEMMA 16. If $\psi$ is obtained from $\phi$ by proper substitution of $\zeta$ for $\alpha$ and $\beta$ does not occur in $\zeta$, then $\vdash^{e} \beta \wedge \pi[\pi(\beta) \rightarrow \pi(\zeta)] \rightarrow\left(\bigwedge^{e} \alpha \phi \rightarrow \psi\right)$

Proof. Lemma 16 follows directly from Lemmas 13, 14, 3 and 12. (Q.E.D.)

LEMMA 17. If ' $\psi^{\prime}$ is obtained from $\psi$ by replacing an occurrence of $\phi$ by an occurrence of $\phi^{\prime}, \vdash \phi \rightarrow \phi^{\prime}$ and $\vdash \phi^{\prime} \rightarrow \phi$, then $\vdash \psi \rightarrow \psi^{\prime}$ and $\vdash \psi^{\prime} \rightarrow \psi$.

Proof. By a simple inductive argument using Lemmas 1-3, 6, 7, (A4)-(A5) and (A13). (Q.E.D.)

LEMMA 18. $\vdash \bigwedge^{e} \alpha \bigvee^{e} \beta \wedge \pi[\pi(\beta) \rightarrow \pi(\alpha)]$

ProOF.

$$
\vdash \bigwedge \beta\left[\bigwedge \pi(\pi(\beta) \rightarrow \pi(\alpha)) \rightarrow \bigwedge^{e} \pi \sim \pi(\beta)\right] \rightarrow\left[\bigwedge \pi(\pi(\alpha) \rightarrow \pi(\alpha)) \rightarrow \AA^{i} \pi \sim \pi(\alpha)\right]
$$

by Lemma 12 ,

$\vdash \bigwedge \beta\left[\bigwedge \pi(\pi(\beta) \rightarrow \pi(\alpha)) \rightarrow \bigwedge^{e} \pi \sim \pi(\beta)\right] \rightarrow \bigwedge^{e} \pi \sim \pi(\alpha)$

by Lemmas 1, 2 and 3,

$\vdash \stackrel{\ell}{\beta} \sim \wedge \pi[\pi(\beta) \rightarrow \pi(\alpha)] \rightarrow \stackrel{\complement}{\wedge} \pi \sim \pi(\alpha)$

by (A13) and Lemmas 17, 1 and 2, and therefore

$$
\vdash \stackrel{e}{\wedge} \alpha \stackrel{e}{\bigvee} \beta \wedge \pi[\pi(\beta) \rightarrow \pi(\alpha)]
$$

by Lemmas 3, 1, 2 and (A13). (Q.E.D.)

Lemma 18 establishes that $\vdash \bigwedge^{e} \alpha \bigvee^{e} \beta \alpha \equiv \beta$. This formula (as a first order formula) was taken as an axiom in the first order logic (with identity) of actual and possible objects formulated in [2] and [3]. Accordingly, as modus ponens is the only inference rule of that first order system and as each of its axioms is either an axiom or derivable (by Lemmas 8, 9 and 18) as a theorem of the present system, then every (second order analogue of a) theorem of the first order logic (with identity) of actual and possible objects is a theorem of second order logic with e-attributes.

For the statement of the lemmas to follow we not only retain our convention of using specific groups of Greek letters for the different kinds of expressions but assume in addition that $n$ is a natural number, $\pi$ is an $n$-place predicate variable, and $\alpha_{0}, \cdots, \alpha_{n-1}$ are pairwise distinct individual variables.

We remark that Lemmas 19 and 20 are the analogues of Lemmas 10 and 11 . The analogue of Lemma 12, viz., the general specification principle for attributes, is provable in the present system but as it is not needed to establish our completeness theorem we avoid giving its proof here. Lemma 21 is a restricted analogue of Lemma. 13. A more general version of the specification principle for e-attributes is provable, but, again, as it is not needed here we avoid giving its proof. The proofs for Lemmas 19 and 20 are similar to the proofs of their analogues in the system of [6].

LEMMA 19. If $\alpha_{0}, \cdots, \alpha_{n-1}$ are all the individual variables that have free occurrences in $\psi$, then $\vdash \wedge \alpha_{0} \cdots \wedge \alpha_{n-1}\left[\pi\left(\alpha_{0}, \cdots, \alpha_{n-1}\right) \leftrightarrow \psi\right] \rightarrow\left(\phi \rightarrow \phi\left[\begin{array}{c}\pi\left(\alpha_{0}, \cdots, \alpha_{n-1}\right) \\ \psi\end{array}\right]\right)$ and $\vdash \wedge \alpha_{0} \cdots \wedge \alpha_{n-1}\left[\pi\left(\alpha_{0}, \cdots, \alpha_{n-1}\right) \leftrightarrow \psi\right] \rightarrow\left(\phi\left[\begin{array}{c}\pi\left(\alpha_{0}, \cdots, \alpha_{n-1}\right) \\ \psi\end{array}\right] \rightarrow \phi\right)$.

LEMMA 20. If $\alpha_{0}, \cdots, \alpha_{n-1}$ are all the individual variables that have free occur- 
rences in $\psi$, and $\pi$ does not have a free occurrence in $\phi\left[\begin{array}{c}\pi\left(\alpha_{0}, \cdots, \alpha_{n-1}\right) \\ \psi\end{array}\right]$, then $\vdash \wedge \pi \phi \rightarrow \phi\left[\begin{array}{c}\pi\left(\alpha_{0}, \cdots, \alpha_{n-1}\right) \\ \psi\end{array}\right]$.

LEMMA 21. If $\alpha_{0}, \cdots, \alpha_{n-1}$ are all the individual variables that have free occurrences in $\psi$, and $\pi$ does not have a free occurrence in $\phi\left[\begin{array}{c}\pi\left(\alpha_{0}, \cdots, \alpha_{n-1}\right) \\ \psi\end{array}\right]$, then $\vdash^{e} \pi \wedge \alpha_{0} \cdots \wedge \alpha_{n-1}\left[\pi\left(\alpha_{0}, \cdots, \alpha_{n-1}\right) \leftrightarrow \psi\right] \rightarrow\left(\bigwedge^{e} \pi \phi \rightarrow \phi\left[\begin{array}{c}\pi\left(\alpha_{0}, \cdots, \alpha_{n-1}\right) \\ \psi\end{array}\right]\right)$

Proof. Assume the hypothesis. Then,

$\begin{aligned} & \vdash \wedge \alpha_{0} \cdots \wedge \alpha_{n-1}\left[\pi\left(\alpha_{0}, \cdots, \alpha_{n-1}\right) \leftrightarrow \psi\right] \rightarrow\left(\phi \rightarrow \phi\left[\begin{array}{c}\left.\left.\pi\left(\alpha_{0}, \cdots, \alpha_{n-1}\right)\right]\right) \\ \psi\end{array}\right]\right. \\ & \text { by Lemma 19, } \\ & \vdash \wedge \pi\left[\sim \phi\left[\begin{array}{c}\pi\left(\alpha_{0}, \cdots, \alpha_{n-1}\right) \\ \psi\end{array}\right] \rightarrow\left(\phi \rightarrow \sim \wedge \alpha_{0} \cdots \wedge \alpha_{n-1}\left[\pi\left(\alpha_{0}, \cdots, \alpha_{n-1}\right) \leftrightarrow \psi\right]\right)\right]\end{aligned}$

$\vdash \stackrel{e}{\wedge} \sim \phi\left[\begin{array}{c}\pi\left(\alpha_{0}, \cdots, \alpha_{n-1}\right) \\ \psi\end{array}\right]$

by Lemmas 1,2 , and 6 ,

$\rightarrow\left(\bigwedge_{\pi \phi \rightarrow}^{e} \pi \sim \wedge \alpha_{0} \cdots \wedge \alpha_{n-1}\left[\pi\left(\alpha_{0}, \cdots, \alpha_{n-1}\right) \leftrightarrow \psi\right]\right)$

by (A9) and Lemmas 1 and 2

$\vdash \sim \phi\left[\begin{array}{c}\pi\left(\alpha_{0}, \cdots, \alpha_{n-1}\right) \\ \psi\end{array}\right] \rightarrow \stackrel{e}{\Lambda} \sim \phi\left[\begin{array}{c}\pi\left(\alpha_{0}, \cdots, \alpha_{n-1}\right) \\ \psi\end{array}\right]$

by Lemma 7 , and therefore

$\vee^{e} \pi \wedge \alpha_{0} \cdots \wedge \alpha_{n-1}\left[\pi\left(\alpha_{0}, \cdots, \alpha_{n-1}\right) \leftrightarrow \psi\right] \rightarrow\left(\stackrel{e}{\bigwedge} \pi \phi \rightarrow \phi\left[\begin{array}{c}\pi\left(\alpha_{0}, \cdots, \alpha_{n-1}\right) \\ \psi\end{array}\right)\right.$

by Lemmas 1 and 2. (Q.E.D.)

LEMMA 22. If $\pi$ has no free occurrences in $\phi$ and $\alpha_{0}, \cdots, \alpha_{n-1}$ are all the individual variables that have free occurrences in $\phi$, then

$\vdash \wedge \alpha_{0} \cdots \wedge \alpha_{n-1}\left[\phi \rightarrow \bigvee^{e} \pi \pi\left(\alpha_{0}, \cdots, \alpha_{n-1}\right)\right]$

$$
\rightarrow \bigvee^{e} \pi \wedge \alpha_{0} \cdots \wedge \alpha_{n-1}\left[\pi\left(\alpha_{0}, \cdots, \alpha_{n-1}\right) \leftrightarrow \phi\right]
$$

Proof. Assume the hypothesis and let $\sigma$ be an $n$-place predicate variable distinct from $\pi$ and which has no free occurrences in $\phi$. Then,

$$
\begin{aligned}
& \vdash^{e} \sigma \wedge \alpha_{0} \cdots \wedge \alpha_{n-1}\left[\sigma\left(\alpha_{0}, \cdots, \alpha_{n-1}\right) \leftrightarrow \bigvee^{e} \pi \pi\left(\alpha_{0}, \cdots, \alpha_{n-1}\right)\right] \\
& \text { by (A12), } \\
& \vdash \bigwedge^{e} \sigma \bigvee^{e} \pi \wedge \alpha_{0} \cdots \wedge \alpha_{n-1}\left[\pi\left(\alpha_{0}, \cdots, \alpha_{n-1}\right) \leftrightarrow \sigma\left(\alpha_{0}, \cdots, \alpha_{n-1}\right) \wedge \phi\right] \\
& \text { by (A11), } \\
& \vdash^{e} \pi \wedge \alpha_{0} \cdots \wedge \alpha_{n-1}\left[\pi\left(\alpha_{0}, \cdots, \alpha_{n-1}\right) \leftrightarrow \bigvee^{e} \pi \pi\left(\alpha_{0}, \cdots, \alpha_{n-1}\right) \wedge \phi\right]
\end{aligned}
$$

by Lemmas 21 and 2 ,

$$
\begin{aligned}
\vdash \wedge \alpha_{0} \cdots \wedge \alpha_{n-1}\left[\pi\left(\alpha_{0}, \cdots, \alpha_{n-1}\right)\right. & \left.\leftrightarrow \bigvee^{e} \pi \pi\left(\alpha_{0}, \cdots, \alpha_{n-1}\right) \wedge \phi\right] \\
\rightarrow\left(\bigwedge \alpha_{0} \cdots \wedge \alpha_{n-1}[\phi\right. & \left.\rightarrow \bigvee \pi \pi\left(\alpha_{0}, \cdots, \alpha_{n-1}\right)\right] \\
& \left.\rightarrow \wedge \alpha_{0} \cdots \wedge \alpha_{n-1}\left[\pi\left(\alpha_{0}, \cdots, \alpha_{n-1}\right) \leftrightarrow \phi\right]\right)
\end{aligned}
$$

by Lemma 3, (A4) and Lemmas 1 and 2, and therefore 


$$
\begin{aligned}
& \vdash \wedge \alpha_{0} \cdots \wedge \alpha_{n-1}\left[\phi \rightarrow \bigvee^{e} \pi \pi\left(\alpha_{0}, \cdots, \alpha_{n-1}\right)\right] \\
& \rightarrow \bigvee^{e} \pi \wedge \alpha_{0} \cdots \wedge \alpha_{n-1}\left[\pi\left(\alpha_{0}, \cdots, \alpha_{n-1}\right) \leftrightarrow \phi\right] \\
& \text { by Lemmas 6, 7, (A9) and Lemmas } 1 \text { and } \\
& \text { 2. (Q.E.D.) }
\end{aligned}
$$

LEMMA 23. If $\pi$ has no free occurrences in $\phi, \rho$ is a 1-place predicate variable distinct from $\pi$ and which has no free occurrences in $\phi$, and $\alpha_{0}, \cdots, \alpha_{n-1}$ are all the individual variables that have free occurrences in $\phi$, then for each natural number $i<n, \vdash \bigvee^{e} \pi \wedge \alpha_{0} \cdots \wedge \alpha_{n-1}\left[\pi\left(\alpha_{0}, \cdots, \alpha_{n-1}\right) \leftrightarrow \phi\right] \rightarrow \wedge \alpha_{0} \cdots \wedge \alpha_{n-1}\left[\phi \rightarrow \bigvee^{e} \rho\left(\alpha_{1}\right)\right]$

Proof. Assume the hypothesis. Then,

$$
\begin{aligned}
\mathscr{V}^{e} \pi \wedge \alpha_{0} \cdots & \wedge \alpha_{n-1}\left[\pi\left(\alpha_{0}, \cdots, \alpha_{n-1}\right) \leftrightarrow \phi\right] \\
& \rightarrow\left(\bigwedge^{e} \pi \vee \rho \wedge \alpha_{i}\left[\rho\left(\alpha_{i}\right) \leftrightarrow \pi\left(\alpha_{0}, \cdots, \alpha_{n-1}\right) \wedge \phi\right]\right. \\
& \left.\rightarrow \bigvee^{e} \rho \wedge \alpha_{i}\left[\rho\left(\alpha_{i}\right) \leftrightarrow \phi \wedge \phi\right]\right)
\end{aligned}
$$

by Lemma 21 ,

$$
\begin{aligned}
& H^{e} \pi \wedge \alpha_{0} \cdots \wedge \alpha_{n-1}\left[\pi\left(\alpha_{0}, \cdots, \alpha_{n-1}\right) \leftrightarrow \phi\right] \rightarrow \bigvee^{e} \rho \wedge \alpha_{i}\left[\rho\left(\alpha_{i}\right) \leftrightarrow \phi\right] \\
& \text { by (A11) and Lemmas 17, } 1 \text { and 2, } \\
& \mathscr{V}^{e} \rho \wedge \alpha_{i}\left[\rho\left(\alpha_{i}\right) \leftrightarrow \phi\right] \rightarrow\left[\phi \rightarrow \dot{\vee} \rho \rho\left(\alpha_{i}\right)\right]
\end{aligned}
$$

by Lemmas 12, 6 (A9), and Lemmas 7 , 1 and 2 and therefore

$$
\begin{array}{r}
^{e} \pi \wedge \alpha_{0} \cdots \wedge \alpha_{n-1}\left[\pi\left(\alpha_{0}, \cdots, \alpha_{n-1}\right) \leftrightarrow \phi\right] \rightarrow \wedge \alpha_{0} \cdots \wedge \alpha_{n-1}\left[\phi \rightarrow \bigvee_{\rho p}\left(\alpha_{1}\right)\right] \\
\text { by Lemma 3, (A4), (A5) and Lemmas } 1 \\
\text { and 2. (Q.E.D.) }
\end{array}
$$

§5. A completeness theorem for second order logic with e-attributes. The proof we give for the completeness theorem to follow is a natural extension of Henkin's proof for the completeness of standard second order logic. As we are dealing with a wider notion of formulahood, however, it is requisite that we indicate the major steps of the proof.

As usual, we say that a set $\Gamma$ of formulas is consistent if there is no formula $\phi$ such that $\Gamma \vdash \phi$ and $\Gamma \vdash \sim \phi$, and where $L$ is a language, we say that $\Gamma$ is a maximally consistent set of sentences of $L$ if $\Gamma$ is a consistent set of sentences of $L$ such that for each sentence $\phi$ of $L$, either $\phi \in \Gamma$ or $\Gamma \cup\{\sim \phi\}$ is not consistent.

THEOREM 2. If $\Gamma$ is a consistent set of sentences of a language $L$, then there exists a normal secondary model $\mathfrak{B}$ such that every sentence in $\Gamma$ is true in $\mathfrak{B}$.

Proof. Where $\lambda$ is the least infinite ordinal equinumerous with or greater than $L$, we add to $L$ new individual constants $\zeta_{0}, \cdots, \zeta_{\mu}, \cdots(\mu \in \lambda)$ and, for each $n \in \omega$, the $n$-place predicate constants $\pi_{0}^{n}, \cdots, \pi_{\mu}^{n}, \cdots(\mu \in \lambda)$ and call the resulting language $L_{*}$. Let $\Sigma_{1}, \Sigma_{2}, \cdots, \Sigma_{\mu}, \cdots(\mu \in \lambda)$ be an ordering of the set of sentences $\phi$ of $L_{*}$ for which there are an $n \in \omega$, an $n$-place predicate variable $\sigma$, an individual variable $\alpha$ and a formula $\psi$ of $L_{*}$ such that $\phi$ is either $\bigvee^{e} \alpha \psi, \bigvee \propto \psi, \bigvee \sigma \psi$, or $\bigvee^{e} \sigma \psi$ We define by ordinal recursion the chain $\Gamma_{0}, \cdots, \Gamma_{\mu}, \cdots(\mu \in \lambda)$ as follows: (1) $\Gamma_{0}=\Gamma$; (2) if $\bigcup_{\mu \in v} \Gamma_{\mu} \cup\left\{\Sigma_{v}\right\}$ is not consistent, then $\Gamma_{v}=\bigcup_{\mu \in v} \Gamma_{\mu}$; (3) if $\bigcup_{\mu \in v} \Gamma_{\mu} \cup\left\{\Sigma_{v}\right\}$ is consistent, then 
(a) if $\Sigma_{v}=\bigvee \alpha \phi$, for some formula $\phi$ of $L_{*}$ and some individual variable $\alpha$, then $\Gamma_{v}=\bigcup_{\mu \in v} \Gamma_{\mu} \cup\left\{\phi\left[\begin{array}{l}\alpha \\ \zeta_{l}\end{array}\right]\right\}$, where $\iota$ is the least ordinal $k<\lambda$ such that $\zeta_{k}$ does not occur in any member of $\bigcup_{\mu e v} \Gamma_{\mu}$;

(b) if $\Sigma_{v}=V^{e} \alpha \phi$, for some formula $\phi$ of $L_{*}$ and some individual variable $\alpha$, then $\Gamma_{v}=\bigcup_{\mu \in v} \Gamma_{\mu} \cup\left\{\phi\left[\begin{array}{l}\alpha \\ \zeta_{l}\end{array}\right], \vee^{e} \tau \tau\left(\zeta_{l}\right)\right\}$, where $\tau$ is the first 1-place predicate variable and $\iota$ is the least ordinal $k<\lambda$ such that $\zeta_{k}$ does not occur in any member of $\bigcup_{\mu \in v} \Gamma_{\mu}$;

(c) If $\Sigma_{v}=\bigvee \sigma \phi$, for some formula $\phi$ of $L_{*}$, some $n \in \omega$, and some $n$-place predicate variable $\sigma$, then $\Gamma_{v}=\cup_{\mu \in v} \Gamma_{\mu} \cup\left\{\phi\left[\begin{array}{c}\sigma \\ \pi_{\iota}^{n}\end{array}\right]\right\}$, where $\iota$ is the least ordinal $k$ $<\lambda$ such that $\pi_{k}^{n}$ does not occur in any member of $\bigcup_{\mu \in v} \Gamma_{\mu}$; and

(d) If $\Sigma_{v}=\bigvee^{e} \sigma \phi$, for some formula $\phi$ of $L_{*}$, some $n \in \omega$, and some $n$-place predicate variable $\sigma$, then

$\Gamma_{v}=\bigcup_{\mu \in v} \Gamma_{\mu} \cup\left\{\phi\left[\begin{array}{c}\sigma \\ \pi_{t}^{n}\end{array}\right], \stackrel{e}{\vee} \rho \wedge \alpha_{0} \cdots \wedge \alpha_{n-1}\left[\rho\left(\alpha_{0}, \cdots, \alpha_{n-1}\right) \leftrightarrow \pi_{\iota}^{n}\left(\alpha_{0}, \cdots, \alpha_{n-1}\right)\right]\right\}$ where $\rho$ is the first $n$-place predicate variable different from $\sigma, \alpha_{0}, \cdots, \alpha_{n-1}$ are the first $n$ individual variables, and $\iota$ is the least ordinal $k<\lambda$ such that $\pi_{k}^{n}$ does not occur in any member of $\bigcup_{\mu \in v} \Gamma_{\mu}$.

For each $v \in \lambda, \Gamma_{v}$ is consistent. The proof of this is by induction and we must consider four cases. In case $\Sigma_{v}=\bigvee \propto \phi$, for some formula $\phi$ and some individual variable $\alpha$, or $\Sigma_{v}=\bigvee \sigma \phi$, for some formula $\phi$, some $n \in \omega$ and some $n$-place predicate variable $\sigma$, the consistency of $\Gamma_{v}$ follows by the inductive hypothesis and Lemma 4. In case $\Sigma_{v}=\bigvee^{e} \alpha \phi$, for some formula $\phi$ and some individual variable $\alpha$, the consistency of $\Gamma_{v}$ follows by Lemma 4 together with (A13). Finally, in case $\Sigma_{v}=$ $\vee^{e} \sigma \phi$, for some formula $\phi$, some $n \in \omega$, and some $n$-place predicate variable $\sigma$, the consistency of $\Gamma_{v}$ requires Lemma 5, (A9) and (A10), together with Lemma 11 and (A11).

We conclude that $\Gamma_{v}$ is consistent for all $v \in \lambda$. Now let $\Gamma^{\prime}$ be the union of all $\Gamma_{v}$, for $v \in \lambda$, i.e., let $\Gamma^{\prime}=\bigcup_{v \in \lambda} \Gamma_{v}$. In the usual manner, we conclude that $\Gamma^{\prime}$ is consistent, since otherwise $\Gamma_{v}$ is not consistent for some $v \in \lambda$, which is impossible. Accordingly, by Lindenbaum's lemma, there is a maximally consistent set $K$ of sentences of $L_{*}$ such that $\Gamma^{\prime} \subseteq K$.

By a closed term of $L_{*}$ we shall mean a term of $L_{*}$ in which no (individual) variable occurs. Where $\eta$ is a closed term of $L_{*}$, let $[\eta]$ be the set of closed terms $\zeta$ of $L_{*}$ such that for some 1-place predicate variable $\pi, \bigwedge \pi[\pi(\eta) \rightarrow \pi(\zeta)] \in K$.

Now let $f$ be that function whose domain is the set of predicate constants of $L_{*}$ and which is such that for each natural number $n$ and for each $n$-place predicate constant $\pi$ of $L_{*}, f(\pi)=$ the set of $n$-tuples $\left\langle\left[\eta_{0}\right], \cdots,\left[\eta_{n-1}\right]\right\rangle$ such that $\eta_{0}, \cdots, \eta_{n-1}$ are closed terms of $L_{*}$ and $\pi\left(\eta_{0}, \cdots, \eta_{n-1}\right) \in K$.

We proceed by constructing an appropriate normal 2-model. Let $A$ be the set of [ $\eta$ ] such that $\eta$ is a closed term of $L_{*}$ and for some 1-place predicate variable $\pi$, $\dot{V} \pi \pi(\eta) \in K$. Let $B$ be the set of $[\eta]$ such that $\eta$ is a closed term of $L_{*}$, and let $R$ be 
the function whose domain is $L_{*}$ and which is such that for all $n \in \omega:$ (1) for all $n$-place predicate constants $\pi$ in $L_{*}, R(\pi)=f(\pi)$, and (2) for all $n$-place operation constants $\delta$ of $L_{*}, R(\delta)$ is that function the domain of which is $B^{n}$ and which is such that for all $\left\langle\left[\zeta_{0}\right], \cdots,\left[\zeta_{n-1}\right]\right\rangle \in B^{n}, R(\delta)\left(\left\langle\left[\zeta_{0}\right], \cdots,\left[\zeta_{n-1}\right]\right\rangle\right)=\left[\delta\left(\zeta_{0}, \cdots, \zeta_{n-1}\right)\right]$. We note that by definition of $A, B$ and $R,\langle A, B, R\rangle$ is an $L_{*}$-model.

Consider now the $\omega$-termed sequence $\left\langle\mathbf{F}_{n}\right\rangle_{n \in \omega}$ which is such that for each $n \in \omega$ $\mathbf{F}_{n}$ is the family of sets $f(\pi)$ where $\pi$ is an $n$-place predicate constant of $L_{*}$. We set $\mathfrak{B}=\left\langle\langle A, B, R\rangle,\left\langle\mathbf{F}_{n}\right\rangle_{n \in \omega}\right\rangle$ and observe that by definition $\mathfrak{B}$ is a secondary $L_{*}$-model.

We show now by recursion: for all assignments $a$ in $\mathscr{B}$ and for all closed terms $\eta$ of $L_{*},[\eta]=\operatorname{Val}(\eta, \mathfrak{B}, a)$. Suppose $a$ is an assignment in $\mathfrak{B}$ and that $\eta$ is an individual constant of $L_{*}$, i.e., $\eta$ is a 0 -place operation constant of $L_{*}$. Then $\operatorname{Val}(\eta, \mathfrak{B}, a)=$ $R(\eta)(0)=[\eta]$. Suppose now that $\delta\left(\eta_{0}, \cdots, \eta_{n-1}\right)$ is a closed term of $L_{*}$. Then, by the inductive hypothesis, $\operatorname{Val}\left(\eta_{i}, \mathfrak{B}, a\right)=\left[\eta_{t}\right]$, for all $i<n$. By definition, $\operatorname{Val}\left(\delta\left(\eta_{0}, \cdots, \eta_{n-1}\right), \mathfrak{B}, a\right)$

$$
=R(\delta)\left(\left\langle\operatorname{Val}\left(\eta_{0}, \mathfrak{O}, a\right), \cdots, \operatorname{Val}\left(\eta_{n-1}, \mathfrak{B}, a\right)\right\rangle\right)=\left[\delta\left(\eta_{0}, \cdots, \eta_{n-1}\right)\right] .
$$

Accordingly, the above claim that $[\eta]=\operatorname{Val}(\eta, \mathfrak{B}, a)$ for all assignments $a$ in $\mathscr{B}$ and all closed terms $\eta$ of $L_{*}$ holds.

Where $a$ is an assignment in $\mathfrak{B}$ and $\phi$ is a formula of $L_{*}$, let $\{\phi\}_{a}$ be the set of formulas $\psi$ of $L_{*}$ "such that for some natural numbers $n$ and $k$, there are pairwise distinct individual variables $\alpha_{0}, \cdots, \alpha_{n-1}$, distinct predicate variables $\sigma_{0}, \cdots, \sigma_{k-1}$, closed terms $\eta_{0}, \cdots, \eta_{n-1}$ of $L_{*}$ and predicate constants $\rho_{0}, \cdots, \rho_{k-1}$ of $L_{*}$ such that for all natural numbers $i<n, a\left(\alpha_{i}\right)=\left[\eta_{i}\right]$ and for all natural numbers $j<k$, $a\left(\sigma_{j}\right)=f\left(\rho_{j}\right)$ (and $\rho_{j}$ is therefore a predicate constant of the same number of places as $\sigma_{j}$ ), and $\psi=\phi\left[\begin{array}{lll}\alpha_{0} & \cdots & \alpha_{n-1} \\ \eta_{0} & \cdots & \eta_{n-1}\end{array}\right]\left[\begin{array}{lll}\sigma_{0} & \cdots & \sigma_{k-1} \\ \rho_{0} & \cdots & \rho_{k-1}\end{array}\right]$. We note that if $a$ is an assignment in $\mathfrak{B}$ and $\phi$ is a sentence of $L_{*}$, then $\{\phi\}_{a}=\{\phi\}$.

Let $M$ be the set of formulas $\phi$ of $L_{*}$ such that for all assignments $a$ in $\mathscr{B}$ and for all sentences $\psi$ in $\{\phi\}_{a}, a$ satisfies $\phi$ in $\mathfrak{B}$ if and only if $\psi \in K$. It can be shown that every formula of $L_{*}$ is in $M$. For instance, from the definition of satisfaction in a 2-model and the fact that $K$ is a maximally consistent set of sentences of $L_{*}$, it follows that $(\phi \rightarrow \psi)$ belong to $M$ whenever $\phi, \psi \in M$. Moreover, $\wedge \mu \phi$ is in $M$ by either Lemma 12 or Lemma 20, while $\bigwedge^{e} \mu \phi$ is in $M$ by Lemma 13 if $\mu$ is an individual variable, and, if $\mu$ is a predicate variable, by (A13) and Lemmas 21,22 and 23.

Since every formula of $L_{*}$ is in $M$ and since $\{\phi\}_{a}=\{\phi\}$, where $a$ is an assignment in $\mathfrak{B}$ and $\phi$ is a sentence of $L_{*}$, then for each sentence $\phi$ of $L_{*}, \phi$ is true in $\mathfrak{B}$ if and only if $\phi \in K$. Accordingly, since $\Gamma \subseteq K$, then every sentence in $\Gamma$ is true in $\mathscr{B}$.

It remains only to show that $\mathfrak{B}$ is normal. But if $\phi$ is a formula of $L_{*}$ which is an instance of (A6) and $a$ is any assignment in $\mathfrak{B}$, then, since $K$ is maximally consistent, every sentence in $\{\phi\}_{a}$ is an axiom and therefore in $K$; and, accordingly, $a$ satisfies $\phi$ in $\mathfrak{B}$; that is, $\phi$ is true in $\mathfrak{B}$. Therefore $\mathfrak{B}$ is normal. (Q.E.D.)

THEOREM 3. If $\phi$ is a secondarily valid formula, then $\phi$ is a theorem of second order logic with e-attributes.

Proof. Assume that $\phi$ is 2 -valid and, by reductio, that $\phi$ is not a theorem of second order logic with e-attributes. Let $\alpha_{0}, \cdots, \alpha_{n-1}$ be all the individual variables 
that have free occurrences in $\phi$; let $\pi_{0}, \cdots, \pi_{k-1}$ be all the predicate variables that have free occurrences in $\phi$; and let $\sigma_{0}, \cdots, \sigma_{k-1}$ be the first $k$ predicate variables which do not occur in $\phi$ and which are such that $\sigma_{i}$ is of the same number of places as $\pi_{i}$, for all $i<k$. Let $\psi=\wedge \sigma_{0} \cdots \wedge \sigma_{k-1} \wedge \alpha_{0} \cdots \wedge \alpha_{n-1} \phi\left[\begin{array}{lll}\pi_{0} & \cdots & \pi_{k-1} \\ \sigma_{0} & \cdots & \sigma_{k-1}\end{array}\right]$. Then $\psi$ is not a theorem, since otherwise, by Lemmas 20 and $12, \phi$ is a theorem which, by assumption it is not. Accordingly, $\{\sim \psi\}$ is consistent, and therefore, by Theorem 2 , there exists a normal 2 -model in which $\sim \psi$ is true. But then, by definition of satisfaction, $\phi$ is not 2-valid, which is impossible. (Q.E.D.)

\section{REFERENCES}

[1] A. ChURCH, Introduction to mathematical logic, vol. 1, Princeton, N.J., 1956.

[2] N. Cocchiarella, Tense logic: A study of temporal reference, Doctoral thesis, UCLA, 1966.

[3] N. Cocchiarella, A logic of actual and possible objects, this Journal, vol. 31 (1966), p. $688 \mathrm{f}$.

[4] N. CocchIarella, A completeness theorem in tense logic, this Journal, vol. 31 (1966), p. 689 .

[5] N. Cocchiarella, Modality within tense logic, this Journal, vol. 31 (1966), p. 890f.

[6] N. Cocchiarella, A substitution free axiom set for second order logic, forthcoming in Notre Dame journal of formal logic.

[7] N. Cocchiarella, Some remarks on second order logic with existence attributes, Noûs, vol. 2 (1968), pp. 165-175.

[8] L. HenkIn, Completeness in the theory of types, this Journal, vol. 15 (1950), pp. 81-91.

[9] A. N. Prior, Past, present and future, Oxford Univ. Press, Oxford, 1967.

INDIANA UNIVERSITY 